\title{
The Types of Tone Sandhi in Chaoyang Dialect
}

\author{
Huang Qiye ${ }^{1, a}$
}

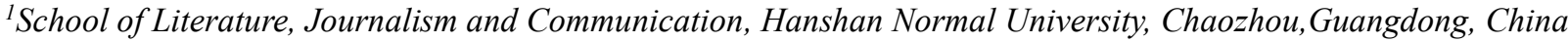 \\ afeliciawong@163.com
}

\begin{abstract}
Chaoyang dialect is a sub-type of the Chaoshan dialect of south Min dialect in Guangdong. Compared with the surrounding areas of Chaoshan, Chaoyang dialect has more differences, especially the tonal system. Its basic tones and sandhi tones are obviously different from the main types of Chaoshan dialects. At the same time, there are also internal differences in Chaoyang area, showing different characteristics. Based on the data obtained from field surveys, this article introduces the tones of Chaoyang dialect in various places, proposes classification standards, sorts out the tones of Chaoyang dialect from two aspects, and elaborates its characteristics, trying to change the tone sandhi of Chaoyang dialect. Make a preliminary comprehensive study of the phenomenon.
\end{abstract}

Keywords: Chaoyang dialect, tone sandhi, tone value

\section{潮阳方言连读变调的类型}

黄绮烨 $1, \mathrm{a}$

${ }^{1}$ 韩山师范学院文学与新闻传播学院, 潮州, 广东, 中国

afeliciawong@163.com

\section{摘要}

潮阳方言是广东闽南方言潮汕话的一种次类型, 与潮汕周边区域相较, 潮阳方言存在较多差异, 特别是声调系 统, 其基本调和连读调均与潮汕方言的主要类型存在明显差异。同时, 潮阳地区内也存在内部差异性, 体现出 不同的特点。本文基于田野调查中所得数据, 介绍潮阳方言各地点的声调情况，提出分类标准，从基本调和连 读调两个方面, 对其进行类型化梳理, 并阐述其特征, 试图对潮阳方言连读变调现象作初步的全面性研究。

关键词: 潮阳方言, 连读变调, 声调调值

\section{1. 前言}

本文所讨论的潮阳方言, 是指旧广东省潮阳市, 即今属广东省汕头市下辖的潮阳区及潮南区 (以下统 称为 “潮阳地区”) 区域内普遍通行的潮汕方言。潮 汕方言, 为闽南方言中的一个次方言, 在语言特色上 共享闽南方言区域内的共同特点，但也存在其独特性。 潮阳地区位于潮汕地区的中间区域, 东连汕头市金平 区、濠江区，西接揭阳市普宁县级市，南邻揭阳市惠 来县, 北界揭阳市揭东区。但潮阳方言却与周边区域 呈现较多明显的差异, 主要表现在语音 (声母、韵母 和声调）和词汇上, 如鱼韵字读为 $[u]$ （其他地区多 为 $[m]$ 或 $[i]$ ), 茄子的词形为 “落苏” (其他地区多为 “茄”)。尤其是声调系统, 其基本调和连读调均与潮 汕方言的主要类型存在较大差异。如林伦伦（1995）
的《潮汕方言声调研究》 ${ }^{[1]}$ 对潮汕地区七种主要方言 的声调系统（包括基本调和连读调）进行描写, 潮阳 方言与其他方言相较，在调值上呈现其独特之处。同 时, 通过调查笔者发现, 潮阳地区内部各地也存在明 显差异。

前人对于潮阳方言声调的深入研究, 主要集中于 张盛裕 $(1979 ， 1980 ， 1981)$ 的《潮阳方言的语音系 统》 ${ }^{[2]}$ 及 《潮阳方言的连读变调》 ${ }^{[3]}$ 、《潮阳方言的连 读变调 (二) 》 ${ }^{[4]}$ 和张屏生的《潮阳话和其他闽南话 的比较》 ${ }^{[5]}$ 。两位作者均对潮阳方言的声调及连读变 调系统进行全面、深刻的描写, 同时列出大量例词, 为本文的研究提供可信、有力的语料依据。但是, 两 位作者的文中均仅描写潮阳方言的一个地点, 张盛裕 文中未说明调查地点, 这可能是潮阳地区的中心地或 作者的出身地; 而张屏生文中说明其调查地点为谷饶 
镇一点。但是对潮阳地区其他地点的情况仍未可知, 我们也未能一窥潮阳方言的全貌。因此, 对潮阳方言 各地点进行全面的方言调查, 有其必要性。

岩田礼（2016）对闽语的连读变调提出三个分类 标准, 并以此总结闽南型（闽南方言）的三个特征: “1. 非末位声调不受到末位声调的制约 (context-free); 2. 以范畴性的链移式交替 (chain shift）为主，但总有一部分声调不加入链移圈; 3 . 倒数第三位及其前的变调与倒数第二位相同。” ${ }^{[6]}$ 潮 汕方言属闽语闽南区的潮汕片, 作为闽南语的一种次 方言, 潮汕方言也具有闽南方言的特征, 特别是特征 1 和特征 2 。本文主要论及特征 2 , 即在潮阳方言多 数地点的声调系统中, 其连读调调值与基本调调值处 于一个系统内, 基本不产生新的调值。

本文基于田野调查中所得数据, 介绍潮阳方言各 地点的声调情况, 提出分类标准, 从基本调和连读调 两个方面, 对其进行类型化梳理, 并阐述其特征, 试 图对潮阳方言连读变调现象作初步的全面性研究。

\section{2. 调查相关说明}

笔者于 2014 年起, 数次前往潮阳地区进行田野 调查。笔者在潮阳地区的调查地点, 共计 18 个。其 中潮阳区 9 个, 分别为关埠镇、西䶻镇、河溪镇、棉 北街道、谷饶镇、贵屿镇、铜孟镇、和平镇和海门镇; 潮南区 9 个, 分别为陈店镇、司马浦镇、峡山街道、 仙城镇、两英镇、胒岗镇、成田镇、陇田镇和井都镇。 区域内多数地点沿练江流域分布。本文中对所论述的 声调调值均以本人田野调查所得数据为材料。

有关调查对象, 原则上 1 个调查点以 1 名发音人 的语音情况作为主要代表, 必要时也参考同一调查点 的其他发音人的语音情况。对发音人的选取, 有如下 要求: 60 岁以上土生土长的男性发音人, 没有 (或 较少) 长期离开本地的生活经历, 能说地道的本地话, 且无发音障碍。对调查对象的职业、学历 (文盲亦可) 不做要求, 以尽量获得全面且第一线的语音材料。

调查方法主要为面对面调查法, 调查时依据事先 准备的调查项目表, 采用直接提问法、图片（实物） 提示法、场景描述法等提问形式对发音人进行询问、 记录并录音存档。关于调查项目表的制作, 主要根据 前人调查的语料, 从中选取具有区域共同性的基本词 汇作为调查项目 (约 180 个)。调查项目主要以双音 节词为主，同时也调查单音节词和多音节词。

本文所使用的声调调值主要通过对双音节词的 提取获得。将潮汕方言中的八个声调进行两两组合, 每个声调均出现在非末位音节 (如 T1+T8、T1+T7、 $\mathrm{T} 1+\mathrm{T} 6 \ldots$ ) 和末位音节 (如 $\mathrm{T} 8+\mathrm{T} 1 、 \mathrm{~T} 7+\mathrm{T} 1 、 \mathrm{~T} 6+\mathrm{T} 1 \ldots$ )， 合计 64 个双音节词。双音节词项目的选择上, 尽量 采用区域内共通使用的日常词, 以期获得发音人自然 的声调调值。同时, 调查记音人均为笔者, 以图尽量 保证所有调查点的声调调值具有统一性, 排除因调查
记录人、调查项目等干扰因素而造成的记音偏差。

\section{3. 潮阳方言的基本调}

以下说明潮阳方言的基本调。关于全文提及的两 个概念, 基本调 (base tone) 为变调前调值, 在潮 汕方言中表现为末位音节的调值。连读调 (sandhi tone）为变调后调值，表现为非末位音节的调值。

表 1 为潮阳方言基本调的两种类型, 其分类标准 主要为声调数量, 其次为各声调调值。

\section{表 1 潮阳方言的基本调}

\begin{tabular}{|c|c|c|c|c|}
\hline \multirow{2}{*}{ 声调调类 } & \multicolumn{3}{|c|}{ 类型 } \\
\cline { 3 - 5 } & $\mathrm{a}-1$ 型 & $\mathrm{a}-2$ 型 & $\mathrm{b}$ 型 \\
\hline $\mathrm{T} 1$ & 阴平 & 33 & 33 & 21 \\
\hline $\mathrm{T} 2$ & 阳平 & 55 & 55 & 55 \\
\hline $\mathrm{T} 3$ & 阴上 & 53 & 53 & 53 \\
\hline $\mathrm{T} 4$ & 阳上 & 35 & 35 & 35 \\
\hline $\mathrm{T} 5$ & 阴去 & 213 & 213 & 31 \\
\hline $\mathrm{T} 6$ & 阳去 & 11 & 31 & 31 \\
\hline $\mathrm{T} 7$ & 阴入 & 2 & 2 & $\underline{32}$ \\
\hline $\mathrm{T} 8$ & 阳入 & 5 & 5 & $\underline{54}$ \\
\hline
\end{tabular}

首先根据声调数量分出两种类型, $\mathrm{a}$ 型 (包括 $\mathrm{a}-1$ 型和 $a-2$ 型) 为八声调, $b$ 型为七声调, 阴去调 (T5) 和阳去调 (T6) 合流为一。其次根据声调调值将 a 型 分为两种次类型, 其区别在于阳去调 (T6) 的调值不 同, a-1 型为低平调 $11, a-2$ 型为中降调 31。同时, 在调值方面, $\mathrm{a}$ 型与 $\mathrm{b}$ 型也存在较大差异, 主要表现 在阴平调（T1）、阴去调（T5）、阳去调（T6）、阴入 调（T7）和阳入调 (T8) 中。

a -1 型和 $a-2$ 型在潮阳地区的分布较少，仅为两 个地点, 即关埠镇 ( $a-1$ 型) 和西䶻镇 ( $a-2$ 型)。从 地理上看, 这两个镇更靠近揭阳市揭东区, 声调上也 共享相同的特点。其余地点 (共 16 个) 均为 $\mathrm{b}$ 型, 这也是潮阳地区区域内的主流类型。

观察以上类型, 我们可得出潮阳方言基本调的特 征:

i. 内部一致性较强, 潮阳地区内的绝大多数地 点均为七声调类的 $b$ 型, 其合流情况均为阴去调 (T5) 和阳去调（T6）归为一类。同时, 在具体基本调调值 的表现上也呈现高度一致性。

ii. 就调型来看, 潮阳方言的主流类型一一b 型 中基本调的降调占明显优势, 分别有阴平调 (T1) 调 值 21、阴上调（T3）调值 53、去声调（T5、T6）调 值 31、阴入调（T7）调值 32 及阳入调（T8）调值 $\underline{54}$ 。

\section{4. 潮阳方言的连读调}

以下具体说明潮阳方言连读调的具体情况。其共 同特征如下：

i . 潮阳方言的连读变调, 基本属于范畴化交替 (categorical alternation), 即变调后的连读调调 
值，属于基本调调值范畴内，并未产生系统内新的调 值。但是，个别地点中仍可见新调值的出现，如谷饶 镇的阳去调（T6）的连读调调值为 11 , 这不在其基 本调（属上文 $b$ 型）的范畴内。

ii. 潮阳方言各地点的连读变调系统, 均可观察 到合流现象的产生, 并且根据合流情况可将其分出三 大类，具体内容将在下文阐述。

iii. 从调型上看, 潮阳方言各地点中的连读调仍 以降调为主, 但同时均可观察到平调化的趋势, 即变 调后的连读调调型中, 平调增多, 分别为低平调 11、 中平调 33 和高平调 55 。

\section{1. 分类标准}

从调类的合流角度出发, 确立了以下三个标准:

I. 阴声调类（阴平 T1、阴上 T3、阴去 T5、阴 入 T7）是否参与声调的合流。

II. 如果阴声调类参与合流, 阴上调 T3 或阴去调 $\mathrm{T} 5$ 是否参与声调的合流。

III. 具体的合流情况。

首先根据标准 I 将 18 个方言点的连读调分出 $\mathrm{A}$ 型和 B、C 型, 其次, 根据标准 II 分出 A、B、C 型三 种类型, 最后, 根据标准III分出各小类, 将 $\mathrm{A} 、 \mathrm{~B} 、 \mathrm{C}$ 型各细分为 $1 、 2$ 次型。

因此, 18 个地点的调值共分出 A-1 型、 A-2 型、 B-1 型、 B-2 型和 C -1 型、 -2 型, 以下分别进行详 细阐述。

\section{2. $A$ 型}

表 2 至表 4 分别为潮阳方言连读调的三种类型。 其中 “/” 表示该调类内出现两种连读调调值, “/” 前的调值出现的次数较多, “/” 后的调值出现的次 数较少。分类时主要以多数情况 (即 “/”前的调值) 为依据。

表 2 潮阳方言的连读调（A 型）

\begin{tabular}{|c|c|c|c|}
\hline \multirow{2}{*}{ 声调调类 } & \multicolumn{2}{|c|}{ 类型 } \\
\cline { 3 - 4 } & $\mathrm{A}-1$ 型 & $\mathrm{A}-2$ 型 \\
\hline $\mathrm{T} 1$ & 阴平 & 33 & 33 \\
\hline $\mathrm{T} 2$ & 阳平 & 11 & 11 \\
\hline $\mathrm{T} 3$ & 阴上 & 35 & $53 / 31$ \\
\hline $\mathrm{T} 4$ & 阳上 & 21 & 21 \\
\hline $\mathrm{T} 5$ & 阴去 & 53 & 55 \\
\hline $\mathrm{T} 6$ & 阳去 & 11 & 11 \\
\hline $\mathrm{T} 7$ & 阴入 & 5 & $\underline{54}$ \\
\hline $\mathrm{T} 8$ & \multicolumn{1}{|c|}{ 阳入 } & 2 & $\underline{32} \mathbf{5 4}$ \\
\hline
\end{tabular}

潮阳地区中 $A$ 型（主要是 $A-2$ 型）的分布最多、 最广, 共 12 个地点。其中, 仅关埠镇一点为 $\mathrm{A}-1$ 型, 而属于 $A-2$ 型的地点有谷饶镇、铜孟镇、和平镇、海 门镇、陈店镇、司马浦镇、仙城镇、两英镇、䶻岗镇、
成田镇和井都镇, 共计 11 点, 具有明显的连续性分 布特征。

$A$ 型的主要特征为:

i . 阴声调类不参与声调的合流。

ii. 阳平调与阳去调合流, 即 $\mathrm{T} 2=\mathrm{T} 6$, 调值为 11 。 连读变调后变为七声调。

A-1 型与 A-2 型的主要区别在于阴上调（T3）、 阴去调（T5）、阴入调（T7）和阳入调（T8）四个声 调的调值。阴上调中, $\mathrm{A}-1$ 型为升调 35, A-2 型为降 调 53 或 31 , 从调型和调值上看, 二者差距较大。阴 去调中, A-1 型为降调 53, A-2 型为平调 55 , 从调型 和调值上看亦有差异。阴入调和阳入调中, $A-1$ 型为 短促调 5 和 $2, A-2$ 型为略降调 54 和 32 或 54 , 从调 型和调值上呈明显差异。

从与基本调的类型的对应上看, $\mathrm{A}-1$ 型对应 $\mathrm{a}^{-1}$ 型, $A-2$ 型对应 $b$ 型。

\section{3. $B$ 型}

\section{表 3 潮阳方言的连读调（B 型）}

\begin{tabular}{|c|c|c|c|}
\hline \multirow{2}{*}{ 声调调类 } & \multicolumn{2}{|c|}{ 类型 } \\
\cline { 3 - 4 } & B-1 型 & B-2 型 \\
\hline $\mathrm{T} 1$ & 阴平 & 33 & 33 \\
\hline $\mathrm{T} 2$ & 阳平 & $53 / 31$ & $53 / 11$ \\
\hline $\mathrm{T} 3$ & 阴上 & 31 & 53 \\
\hline $\mathrm{T} 4$ & 阳上 & 21 & 21 \\
\hline $\mathrm{T} 5$ & 阴去 & 55 & 55 \\
\hline $\mathrm{T} 6$ & 阳去 & 33 & 33 \\
\hline $\mathrm{T} 7$ & 阴入 & $\underline{54}$ & $\underline{\mathbf{5 4}}$ \\
\hline $\mathrm{T} 8$ & 阳入 & $\underline{32} \underline{\mathbf{5 4}}$ & $\underline{32} \underline{\mathbf{5 4}}$ \\
\hline
\end{tabular}

潮阳地区中 $\mathrm{B}$ 型共 3 个地点, 分布比较分散。其 中, 河溪镇为 B-1 型, B-2 型分布于棉北街道、陇田 镇。

$B$ 型的主要特征为:

i . 阴声调类参与声调的合流。

ii. 阴平调与阳去调合流, 即 $\mathrm{T} 1=\mathrm{T} 6$, 调值为 33 。

iii. 部分地点中阳平调与阴上调合流, 即 $\mathrm{T} 2=\mathrm{T} 3$, 合流后调值为 53 或 31 。

B-1 型与 B-2 型在调值上表现较为一致, 其主要 区别在于阳平调（T2）、阴上调（T3）两个声调。阳 平调中, B-1 型的主要调值为 53 , 但也出现另一个降 调 31, B-2 型的主要调值亦为 53, 但存在另一个平 调 11 。通过与 $A$ 型的观察可知, B-1 型与 A-2 型的表 现一致。阴上调中, $A-1$ 型为降调 31, A-2 型为降调 53 , 从调型上看, 二者差异较小。

从与基本调的类型的对应上看, $\mathrm{B}$ 型（包括 $\mathrm{B}-1$ 型和 B-2 型）均对应 $b$ 型。 
4. 4. $C$ 型

\section{表 4 潮阳方言的连读调（C 型）}

\begin{tabular}{|c|c|c|c|}
\hline \multirow{2}{*}{ 声调调类 } & \multicolumn{2}{|c|}{ 类型 } \\
\cline { 3 - 4 } & C-1 型 & C-2 型 \\
\hline $\mathrm{T} 1$ & 阴平 & 33 & 33 \\
\hline $\mathrm{T} 2$ & 阳平 & $11 / 53$ & $11 / 55$ \\
\hline $\mathrm{T} 3$ & 阴上 & 31 & 35 \\
\hline $\mathrm{T} 4$ & 阳上 & $213 / 21$ & 33 \\
\hline $\mathrm{T} 5$ & 阴去 & 55 & 55 \\
\hline $\mathrm{T} 6$ & 阳去 & 33 & 33 \\
\hline $\mathrm{T} 7$ & 阴入 & $\underline{54}$ & 5 \\
\hline $\mathrm{T} 8$ & 阳入 & $\underline{32}$ 年 & 2 \\
\hline
\end{tabular}

潮阳地区中 C 型共 3 个地点, 分布上呈不连续性。 其中, 贵屿镇、峡山街道为 $\mathrm{C}-1$ 型, 西䶻镇为 $\mathrm{C}-2$ 型。

$C$ 型的主要特征为:

i . 阴声调类参与声调的合流。

ii. 阴平调与阳去调合流, 即 $\mathrm{T} 1=\mathrm{T} 6$, 调值为 33 。 $\mathrm{C}-2$ 型中, 阳上调 $\mathrm{T} 4$ 还进一步加入合流, 即 $\mathrm{T} 1=\mathrm{T} 4=\mathrm{T} 6$, 调值均为 33 。

iii. 部分地点还可见阳平调与阴去调合流, 即 $\mathrm{T} 2=\mathrm{T} 5$, 合流后调值为 55 。

C-1 型与 C -2 型的主要区别在于阴上调（T3）、 阳上调（T4）、阴入调 (T7) 和阳入调 (T8) 四个声 调的调值。阴上调中, $C-1$ 型为降调 $31, C-2$ 型为升 调 35, 从调型和调值上看, 二者差异明显。阳上调 中, C-1 型为降调 213 或 $21, C-2$ 型为平调 33 , 从调 型和调值上看均有差异。阴入调和阳入调中, $\mathrm{C}-1$ 型 为短促调 5 和 $2, C-2$ 型为略降调 54 和 32 或 54 , 这 与 A-1 型和 A-2 型的情况一致。同时, 阳平调 (T2) 也有部分地点存在调值差异, 即 $C-1$ 型存在降调 53 , C-2 型存在平调 55 。

从与基本调的类型的对应上看, $\mathrm{C}-1$ 型对应 $\mathrm{b}$ 型, C-2 型对应 a -2 型。

\section{5. 结论}

本文对潮阳地区 18 个方言点的声调情况进行考 察, 从基本调和连读调两方面提出分类标准并进行分 类, 归纳各类型的特征及异同点。本文初步得出如下 结论:

$\mathrm{i}$. 潮阳方言内部, 基本调一致性较强, 连读调 呈现出不同的调值, 主要集中在阳平调、阴上调和阳 去调。同时, 阳上调也有细微差异。从声调调型上看, 无论基本调还是连读调, 降调调型总是占优势。同时 平调化现象明显出现在连读调中。 ii. 无论基本调还是连读调，声调演变均为链移 式交替 (且多为范畴内) 和调位区别的中性化这两种 方式。各类型中的连读调均出现了合流现象。

iii. 我们将基本调各类型与连读调各类型进行对 比，可归纳为表 5 的对应关系。

其中观察可知, $a-1$ 型和 $a-2$ 型分别对应 A- 1 型、 C-2 型, 在本文中各只有 1 个地点, 但这并不表示在 潮汕区域内为特殊类型, 针对这一问题, 将会另行撰 文讨论。潮阳方言中占主导地位的 $b$ 型, 对应 A-2 型、 B-1 型、 B- 2 型和 C -1 型, 可见连读调比基本调的变 化更为复杂, 今后将进一步对其进行深入挖掘。

\section{表 5 基本调各类型与连读调各类型的对应关系}

\begin{tabular}{|c|c|}
\hline 基本调 & 连读调 \\
\hline $\mathrm{a}-1$ 型 & $\mathrm{A}-1$ 型 \\
\hline $\mathrm{a}-2$ 型 & $\mathrm{C}-2$ 型 \\
\hline \multirow{3}{*}{$\mathrm{b}$ 型 } & $\mathrm{A}-2$ 型 \\
\cline { 2 - 2 } & $\mathrm{B}-1$ 型 \\
\cline { 2 - 2 } & $\mathrm{B}-2$ 型 \\
\cline { 2 - 2 } & $\mathrm{C}-1$ 型 \\
\hline
\end{tabular}

\section{项目基金}

本文为广东省哲学社会科学 “十三五”规划 2018 年度学科共建项目《地理语言学视角下的潮阳方言连 读变调研究》(项目批准号：GD18XZW05) 的阶段性成 果之一。

\section{REFERENCES}

[1] Lin, L. (1995) A study on tones in Chaoshan dialect. Studies in Language and Linguistics, No.1: 52-59.

[2] Zhang, S. (1981) The phonetic system of Chaoyang dialect. Dialect, No.1: 27-39.

[3] Zhang, S. (1979) The tone sandhi of Chaoyang dialect. Dialect, No.2: 93-121.

[4] Zhang, S. (1980) The tone sandhi of Chaoyang dialect (second). Dialect, No.2: 123-136.

[5] Zhang, P. (1992) The comparison of Chaoyang dialect with other south Min dialects. Chinese Culture University Publishing.Taipei.

[6] Iwata, R. (2016) The types and the geographical distribution of the south Min dialects tone sandhi. A Diachronic Study of the Tone Sandhi Generation Mechanism in Chinese Dialects, No.1: 10-18. 